\title{
On Fixed Point Theorem of Weak Compatible Maps of Type $(\gamma)$ in Complete Intuitionistic Fuzzy Metric Space
}

\author{
Jong Seo Park* \\ *Department of Mathematic Education, Chinju National University of Education, \\ Jinju 660-756, South Korea
}

\begin{abstract}
In this paper, we give definitions of compatible mappings of type $(\gamma)$ in intuitionistic fuzzy metric space and obtain common fixed point theorem under the conditions of weak compatible mappings of type $(\gamma)$ in complete intuitionistic fuzzy metric space. Our research generalize, extend and improve the results given by Sedghi et.al.[12].
\end{abstract}

Key words : Common fixed point theorem, weak compatible maps of type $(\gamma)$, complete intuitionistic fuzzy metric space.

\section{Introduction}

Zadeh[13] was introduced the concept of fuzzy sets. Fang[1], Kaleva and Seikkala[3], George and Veeramani[2] have introduced the concept of fuzzy metric space for each different methods, and some authors have been improved generalized and extended several properties in this space. Also, Kutukcu et.al.[4] obtained the common fixed points of compatible maps of tyle $(\beta)$ on fuzzy metric spaces, and Sedghi et.al.[12] studied the common fixed point of compatible maps of type $(\gamma)$ in complete fuzzy metric spaces.

Recently, Park[5] and Park et.al.[9] defined the intuitionistic fuzzy metric space. Many authors([7], [8], [9] etc) obtained a fixed point theorems in this space. Also, Park[6], Park et.al.[10] introduced the concept of compatible mappings of type $(\alpha)$ and type $(\beta)$, and obtained common fixed point theorems in intuitionistic fuzzy metric space.

In this paper, we give definitions of compatible mappings of type $(\gamma)$ in intuitionistic fuzzy metric space and obtain common fixed point theorem under the conditions of weak compatible mappings of type $(\gamma)$ in complete intuitionistic fuzzy metric space.

\section{Preliminaries}

Throughout this paper, $\mathbf{N}$ denote the set of all positive integers. Now, we begin with some definitions, properties in intuitionistic fuzzy metric space as following:

Let us recall(see [11]) that a continuous $t-$ norm is a

Manuscript received May. 19, 2010; revised Feb. 3, 2011;

Accepted Feb. 6, 2011.

* This paper is supported by the Chinju National University of Edu -cation Research Fund in 2010 operation $*:[0,1] \times[0,1] \rightarrow[0,1]$ which satisfies the following conditions: (a) $*$ is commutative and associative, (b) $*$ is continuous, (c) $a * 1=a$ for all $a \in[0,1]$, (d) $a * b \leq$ $c * d$ whenever $a \leq c$ and $b \leq d(a, b, c, d \in[0,1])$. Also, a continuous $t$-conorm is a operation $\diamond:[0,1] \times[0,1] \rightarrow$ $[0,1]$ which satisfies the following conditions: (a) $\diamond$ is commutative and associative, (b) $\diamond$ is continuous, (c) $a \diamond 0=a$ for all $a \in[0,1]$, (d) $a \diamond b \geq c \diamond d$ whenever $a \leq c$ and $b \leq d$ $(a, b, c, d \in[0,1])$.

Definition 2.1. ([9])The 5 -tuple $(X, M, N, *, \diamond)$ is said to be an intuitionistic fuzzy metric space if $X$ is an arbitrary set, $*$ is a continuous $t$-norm, $\diamond$ is a continuous $t$-conorm and $M, N$ are fuzzy sets on $X^{2} \times(0, \infty)$ satisfying the following conditions; for all $x, y, z \in X$, such that
(a) $M(x, y, t)>0$,
(b) $M(x, y, t)=1 \Longleftrightarrow x=y$,
(c) $M(x, y, t)=M(y, x, t)$,
(d) $M(x, y, t) * M(y, z, s) \leq M(x, z, t+s)$,
(e) $M(x, y, \cdot):(0, \infty) \rightarrow(0,1]$ is continuous
(f) $N(x, y, t)>0$
(g) $N(x, y, t)=0 \Longleftrightarrow x=y$,
(h) $N(x, y, t)=N(y, x, t)$,
(i) $N(x, y, t) \diamond N(y, z, s) \geq N(x, z, t+s)$,
(j) $N(x, y, \cdot):(0, \infty) \rightarrow(0,1]$ is continuous.

Note that $(M, N)$ is called an intuitionistic fuzzy metric on $X$. The functions $M(x, y, t)$ and $N(x, y, t)$ denote the degree of nearness and the degree of non-nearness between $x$ and $y$ with respect to $t$, respectively.

Let $X$ be an intuitionistic fuzzy metric space. For any $t>0$, the open ball $B(x, r, t)$ with center $x \in X$ and ra- 
dius $0<r<1$ is defined by

$B(x, r, t)=\{y \in X: M(x, y, t)>1-r, N(x, y, t)<r\}$

Let $X$ be an intuitionistic fuzzy metric space. Let $\tau$ be the set of all $A \subset X$ with $x \in A$ if and only if there exist $t>0$ and $0<r<1$ such that $B(x, r, t) \subset A$. Then $\tau$ is a topology on $X$ (induced by the intuitionistic fuzzy metric $(M, N))$. A sequence $\left\{x_{n}\right\} \subset X$ converges to $x$ if and only if $M\left(x_{n}, x, t\right) \rightarrow 1, N\left(x_{n}, x, t\right) \rightarrow 0$ as $n \rightarrow \infty$, for all $t>0$. It is called a Cauchy sequence if for any $0<\epsilon<1$ and $t>0$, there exists $n_{0} \in \mathbf{N}$ such that $M\left(x_{n}, x_{m}, t\right)>1-\epsilon, N\left(x_{n}, x_{m}, t\right)<\epsilon$ for any $m, n \geq n_{0}$. The intuitionistic fuzzy metric space $X$ is said to be complete if every Cauchy sequence is convergent. A subset $A$ of $X$ is said to be F-bounded if there exists $t>0$ and $0<r<1$ such that $M(x, y, t)>1-r, N(x, y, t)<r$ for all $x, y \in A$.

Lemma 2.2. ([7])Let $X$ be an intuitionistic fuzzy metric space. Then $M(x, y, t)$ is nondecreasing, $N(x, y, t)$ is nonincreasing with respect to $t$ for all $x, y \in X$

Definition 2.3. Let $X$ be an intuitionistic fuzzy metric space. Then $M, N$ are said to be continuous on $X^{2} \times$ $(0, \infty)$ if

$$
\begin{aligned}
& \lim _{n \rightarrow \infty} M\left(x_{n}, y_{n}, t_{n}\right)=M(x, y, t), \\
& \lim _{n \rightarrow \infty} N\left(x_{n}, y_{n}, t_{n}\right)=N(x, y, t)
\end{aligned}
$$

whenever a sequence $\left\{\left(x_{n}, y_{n}, t_{n}\right)\right\} \subset X^{2} \times(0, \infty)$ converges to a point $(x, y, t) \in X^{2} \times(0, \infty)$.

Definition 2.4. Let $A$ and $B$ be mappings from an intuitionistic fuzzy metric space $X$ into itself. Then the mappings are said to be weak compatible if they commute at their coincidence point, that is, $A x=B x$ implies that $A B x=B A x$.

Definition 2.5. Let $A$ and $B$ be mappings from an intuitionistic fuzzy metric space $X$ into itself. Then the mappings are said to be compatible if

$$
\begin{aligned}
& \lim _{n \rightarrow \infty} M\left(A B x_{n}, B A x_{n}, t\right)=1, \forall t>0 \\
& \lim _{n \rightarrow \infty} N\left(A B x_{n}, B A x_{n}, t\right)=0
\end{aligned}
$$

whenever $\left\{x_{n}\right\}$ is a sequence in $X$ such that $\lim _{n \rightarrow \infty} A x_{n}=\lim _{n \rightarrow \infty} B x_{n}=x \in X$.

Proposition 2.6. The self mappings $A$ and $B$ of an intuitionistic fuzzy metric space $X$ are compatible, then they are weak compatible

The converse is not true as seen in following example:

Example 2.7. Let $X$ be an intuitionistic fuzzy metric space, where $X=[0,2], *, \diamond$ defined $a * b=\min \{a, b\}$, $a \diamond b=\max \{a, b\}$ for all $a, b \in[0,1]$ and $M(x, y, t)=$ $\frac{t}{t+d(x, y)}, N(x, y, t)=\frac{d(x, y)}{t+d(x, y)}$ for all $t>0$ and $x, y \in X$. Define self maps $A$ and $B$ on $X$ as follows:

$$
\begin{aligned}
& A x=\left\{\begin{array}{l}
2 \text { if } 0 \leq x \leq 1 \\
\frac{x}{2} \text { if } 1<x \leq 2
\end{array}\right. \\
& B x=\left\{\begin{array}{l}
2 \text { if } x=1 \\
\frac{x+3}{5} \text { otherwise }
\end{array}\right.
\end{aligned}
$$

and $x_{n}=2-\frac{1}{2 n}$. Then we have $B 1=2=A 1$ and $B 2=$ $1=A 2$. Also, $B A 2=B 1=2, A B 2=A 1=2(B A 2=$ $A B 2=2)$. thus $A$ and $B$ are weak compatible. Also, since $A x_{n}=\frac{1}{2}\left(2-\frac{1}{2 n}\right)=1-\frac{1}{4 n}, B x_{n}=\frac{1}{5}\left(2-\frac{1}{2 n}+3\right)=$ $1-\frac{1}{10 n}$. Thus $\lim _{n \rightarrow \infty} A x_{n}=1=\lim _{n \rightarrow \infty} B x_{n}$. Furthermore, $B A x_{n}=B\left(1-\frac{1}{4 n}\right)=\frac{1}{5}\left(1-\frac{1}{4 n}+3\right)=\frac{4}{5}-\frac{1}{20 n}$, $A B x_{n}=A\left(1-\frac{1}{10 n}\right)=2$.

Now,

$$
\begin{aligned}
& \lim _{n \rightarrow \infty} M\left(A B x_{n}, B A x_{n}, t\right) \\
= & \lim _{n \rightarrow \infty} M\left(2, \frac{4}{5}-\frac{1}{20 n}, t\right)=\frac{5 t}{5 t+6}, \\
& \lim _{n \rightarrow \infty} N\left(A B x_{n}, B A x_{n}, t\right) \\
= & \lim _{n \rightarrow \infty} N\left(2, \frac{4}{5}-\frac{1}{20 n}, t\right)=\frac{6}{5 t+6} .
\end{aligned}
$$

Hence $A$ and $B$ is not compatible

\section{Weak compatible mappings of $\operatorname{type}(\gamma)$}

Definition 3.1. [7]Let $A$ and $B$ be mappings from an intuitionistic fuzzy metric space $X$ into itself. Then the mappings $A$ and $B$ are said to be compatible maps of type $(\gamma)$ if satisfying:

(i) $A$ and $B$ are compatible, that is,

$$
\begin{aligned}
& \lim _{n \rightarrow \infty} M\left(A B x_{n}, B A x_{n}, t\right)=1, \\
& \lim _{n \rightarrow \infty} N\left(A B x_{n}, B A x_{n}, t\right)=0 \forall t>0
\end{aligned}
$$

whenever $\left\{x_{n}\right\} \subset X$ such that $\lim _{n \rightarrow \infty} A x_{n}=$ $\lim _{n \rightarrow \infty} B x_{n}=x \in X$.

(ii)they are continuous at $x$.

On the other hand, we have

$$
\begin{aligned}
A x & =A\left(\lim _{n \rightarrow \infty} A x_{n}\right)=A\left(\lim _{n \rightarrow \infty} B x_{n}\right) \\
& =\lim _{n \rightarrow \infty} B A x_{n}=B\left(\lim _{n \rightarrow \infty} A x_{n}\right)=B x
\end{aligned}
$$

Definition 3.2. [7]Let $A$ and $B$ be mappings from an intuitionistic fuzzy metric space $X$ into itself. The mappings $A$ and $B$ are said to be weak-compatible of type $(\gamma)$ if $\lim _{n \rightarrow \infty} A x_{n}=\lim _{n \rightarrow \infty} B x_{n}=x$ for some $x \in X$ implies that $A x=B x$. 
Remark 3.3. If self maps $A$ and $B$ of an intuitionistic fuzzy metric space $X$ are compatible of type $(\gamma)$, then they are weak compatible of type $(\gamma)$. But the converse is not true.

Lemma 3.4. [12]Let $X$ be an intuitionistic fuzzy metric space.

(i)If we define $E_{\lambda}: X^{2} \rightarrow[0, \infty)$ by

$E_{\lambda}(x, y)=\inf \{t>0 ; M(x, y, t)>1-\lambda, N(x, y, t)<\lambda\}$ for each $\mu \in(0,1)$ there exists $\lambda \in(0,1)$ such that

$E_{\lambda}\left(x_{1}, x_{n}\right) \leq E_{\lambda}\left(x_{1}, x_{2}\right)+E_{\lambda}\left(x_{2}, x_{3}\right)+\cdots E_{\lambda}\left(x_{n-1}, x_{n}\right)$ for any $x_{1}, x_{2}, \cdots, x_{n} \in X$.

(ii) The sequence $\left\{x_{n}\right\}_{n \in N}$ is convergent in intuitionistic fuzzy metric space $X$ if and only if $E_{\lambda}\left(x_{n}, x\right) \rightarrow 0$. Also, the sequence $\left\{x_{n}\right\}_{n \in N}$ is Cauchy sequence if and only if it is Cauchy sequence with $E_{\lambda}$.

Lemma 3.5. [12]Let $X$ be an intuitionistic fuzzy metric space.

$$
\begin{aligned}
& M\left(x_{n}, x_{n+1}, t\right) \geq M\left(x_{0}, x_{1}, k^{n} t\right), \\
& N\left(x_{n}, x_{n+1}, t\right) \leq N\left(x_{0}, x_{1}, k^{n} t\right)
\end{aligned}
$$

for some $k>1$ and for every $n \in N$. Then sequence $\left\{x_{n}\right\}$ is a Cauchy sequence.

Lemma 3.6. ([7])Let $X$ be an intuitionistic fuzzy metric space. If there exists a number $k \in(0,1)$ such that for all $x, y \in X$ and $t>0$,

$M(x, y, k t) \geq M(x, y, t), N(x, y, k t) \leq N(x, y, t)$,

then $x=y$.

\section{Main Results}

Lemma 4.1. Let $A$ and $B$ be self-mappings of a complete intuitionistic fuzzy metric space $X$ satisfying:

(i)There exists a constant $k \in(0,1)$ such that

$$
\begin{aligned}
& M^{2}(A x, B y, k t) *[M(x, A x, k t) M(y, B y, k t)] \\
& \quad * M^{2}(y, B y, k t)+a M(y, B y, k t) M(x, B y, 2 k t) \\
& \geq[p M(x, A x, t)+q M(x, y, t)] M(x, B y, 2 k t), \\
& N^{2}(A x, B y, k t) \diamond[N(x, A x, k t) N(y, B y, k t)] \\
& \quad \diamond N^{2}(y, B y, k t)+a N(y, B y, k t) N(x, B y, 2 k t) \\
& \leq[p N(x, A x, t)+q N(x, y, t)] N(x, B y, 2 k t)
\end{aligned}
$$

for every $x, y \in X$ and $t>0$, where $0<p, q<1$, $0 \leq a<1$ such that $p+q-a=1$. Then $A$ and $B$ have a unique common fixed point in $X$.
Proof. Let $x_{0} \in X$ be an arbitrary point, there exist $x_{1} \in X$ such that $A x_{0}=x_{1}, B x_{1}=x_{2}$. Inductively, construct the sequences $\left\{x_{n}\right\} \subset X$ such that $x_{2 n+1}=A x_{2 n}$, $x_{2 n+2}=B x_{2 n+1}$ for $n=0,1,2, \cdots$. Then we prove that $\left\{x_{n}\right\}$ is a Cauchy sequence. For $x=x_{2 n}, y=x_{2 n+1}$ by (i) we have

$$
\begin{aligned}
& M^{2}(\left.A x_{2 n}, B x_{2 n+1}, k t\right) \\
& * {\left[M\left(x_{2 n}, A x_{2 n}, k t\right) M\left(x_{2 n+1}, B x_{2 n+1}, k t\right)\right] } \\
& * M^{2}\left(x_{2 n+1}, B x_{2 n+1}, k t\right) \\
&+a M\left(x_{2 n+1}, B x_{2 n+1}, k t\right) M\left(x_{2 n}, B x_{2 n+1}, 2 k t\right) \\
& \geq[\left.M\left(x_{2 n}, A x_{2 n}, t\right)+q M\left(x_{2 n}, x_{2 n+1}, t\right)\right] \\
& \quad \times M\left(x_{2 n}, B x_{2 n+1}, 2 k t\right)
\end{aligned}
$$

and

$$
\begin{array}{rl}
M^{2}( & \left.x_{2 n+1}, x_{2 n+2}, k t\right) \\
\quad & {\left[M\left(x_{2 n}, x_{2 n+1}, k t\right) M\left(x_{2 n+1}, x_{2 n+2}, k t\right)\right]} \\
& * M^{2}\left(x_{2 n+1}, x_{2 n+2}, k t\right) \\
+a & M\left(x_{2 n+1}, x_{2 n+2}, k t\right) M\left(x_{2 n}, x_{2 n+2}, 2 k t\right) \\
\geq[p & \left.M\left(x_{2 n}, x_{2 n+1}, t\right)+q M\left(x_{2 n}, x_{2 n+1}, t\right)\right] \\
& \times M\left(x_{2 n}, x_{2 n+2}, 2 k t\right), \\
N^{2}\left(A x_{2 n}, B x_{2 n+1}, k t\right) & \diamond\left[N\left(x_{2 n}, A x_{2 n}, k t\right) N\left(x_{2 n+1}, B x_{2 n+1}, k t\right)\right] \\
\diamond & N^{2}\left(x_{2 n+1}, B x_{2 n+1}, k t\right) \\
+a N & \left(x_{2 n+1}, B x_{2 n+1}, k t\right) N\left(x_{2 n}, B x_{2 n+1}, 2 k t\right) \\
\leq[p N & \left.\left(x_{2 n}, A x_{2 n}, t\right)+q N\left(x_{2 n}, x_{2 n+1}, t\right)\right] \\
\quad \times N\left(x_{2 n}, B x_{2 n+1}, 2 k t\right)
\end{array}
$$

and

$$
\begin{array}{rl}
N^{2}( & \left.x_{2 n+1}, x_{2 n+2}, k t\right) \\
\diamond & \left.\quad N\left(x_{2 n}, x_{2 n+1}, k t\right) N\left(x_{2 n+1}, x_{2 n+2}, k t\right)\right] \\
& \diamond N^{2}\left(x_{2 n+1}, x_{2 n+2}, k t\right) \\
+a N & N\left(x_{2 n+1}, x_{2 n+2}, k t\right) N\left(x_{2 n}, x_{2 n+2}, 2 k t\right) \\
\leq[p & \left.N\left(x_{2 n}, x_{2 n+1}, t\right)+q N\left(x_{2 n}, x_{2 n+1}, t\right)\right] \\
\quad \times N\left(x_{2 n}, x_{2 n+2}, 2 k t\right) .
\end{array}
$$

Then

$$
\begin{gathered}
M^{2}\left(x_{2 n+1}, x_{2 n+2}, k t\right) \\
\quad *\left[M\left(x_{2 n}, x_{2 n+1}, k t\right) M\left(x_{2 n+1}, x_{2 n+2}, k t\right)\right] \\
+a M\left(x_{2 n+1}, x_{2 n+2}, k t\right) M\left(x_{2 n}, x_{2 n+2}, 2 k t\right) \\
\geq(p+q) M\left(x_{2 n}, x_{2 n+1}, t\right) M\left(x_{2 n}, x_{2 n+2}, 2 k t\right), \\
N^{2}\left(x_{2 n+1}, x_{2 n+2}, k t\right) \\
\quad \diamond\left[N\left(x_{2 n}, x_{2 n+1}, k t\right) N\left(x_{2 n+1}, x_{2 n+2}, k t\right)\right] \\
+a N\left(x_{2 n+1}, x_{2 n+2}, k t\right) N\left(x_{2 n}, x_{2 n+2}, 2 k t\right) \\
\leq(p+q) N\left(x_{2 n}, x_{2 n+1}, t\right) N\left(x_{2 n}, x_{2 n+2}, 2 k t\right) .
\end{gathered}
$$


So

$$
\begin{aligned}
& M\left(x_{2 n+1}, x_{2 n+2}, k t\right)+a M\left(x_{2 n+1}, x_{2 n+2}, k t\right) \\
& \geq(p+q) M\left(x_{2 n}, x_{2 n+1}, t\right), \\
& N\left(x_{2 n+1}, x_{2 n+2}, k t\right)+a N\left(x_{2 n+1}, x_{2 n+2}, k t\right) \\
& \leq(p+q) N\left(x_{2 n}, x_{2 n+1}, t\right) .
\end{aligned}
$$

Therefore

$$
\begin{aligned}
& M\left(x_{2 n+1}, x_{2 n+2}, k t\right) \geq M\left(x_{2 n}, x_{2 n+1}, t\right), \\
& N\left(x_{2 n+1}, x_{2 n+2}, k t\right) \leq N\left(x_{2 n}, x_{2 n+1}, t\right) .
\end{aligned}
$$

Similarly, we also have

$$
\begin{aligned}
& M\left(x_{2 n+2}, x_{2 n+3}, k t\right) \geq M\left(x_{2 n+1}, x_{2 n+2}, t\right), \\
& N\left(x_{2 n+2}, x_{2 n+3}, k t\right) \leq N\left(x_{2 n+1}, x_{2 n+2}, t\right) .
\end{aligned}
$$

For $k \in(0,1)$ if $k_{1}=\frac{1}{k}>1$ and $t=k_{1} t_{1}$, then we have

$$
\begin{aligned}
& M\left(x_{n}, x_{n+1}, t\right) \geq M\left(x_{0}, x_{1}, k_{1}^{n} t_{1}\right), \\
& N\left(x_{n}, x_{n+1}, t\right) \leq N\left(x_{0}, x_{1}, k_{1}^{n} t_{1}\right) .
\end{aligned}
$$

By Lemma 3.5, since $\left\{x_{n}\right\}$ is a Cauchy sequence in $X$ which is complete, $\left\{x_{n}\right\}$ converges to $z$ in $X$. Hence $\lim _{n \rightarrow \infty} A x_{2 n}=\lim _{n \rightarrow \infty} x_{2 n+1}=\lim _{n \rightarrow \infty} x_{2 n+2}=$ $\lim _{n \rightarrow \infty} B x_{2 n+1}=z$.

Now, taking $x=z$ and $y=x_{2 n+1}$ in (i), we have as $n \rightarrow \infty$,

$$
\begin{aligned}
& M^{2}(A z, z, k t) *[M(z, A z, k t) M(z, z, k t)] \\
& * M^{2}(z, z, k t)+a M(z, z, k t) M(z, z, 2 k t) \\
& \geq[p M(z, A z, t)+q M(z, z, t)] M(z, z, 2 k t), \\
& N^{2}(A z, z, k t) \diamond[N(z, A z, k t) N(z, z, k t)] \\
& \diamond N^{2}(z, z, k t)+a N(z, z, k t) N(z, z, 2 k t) \\
& \leq[p N(z, A z, t)+q N(z, z, t)] N(z, z, 2 k t) .
\end{aligned}
$$

Therefore

$$
\begin{aligned}
& M(A z, z, k t)+a \geq p M(z, A z, t)+q, \\
& N(A z, z, k t) \leq 0
\end{aligned}
$$

for all $t>0$, so $A z=z$. Taking $x=x_{2 n}$ and $y=z$ in (i), we have as $n \rightarrow \infty$,

$$
\begin{aligned}
& M(z, B z, t)+a \geq p+q, \\
& N(z, B z, t)+a N(z, B z, t) \leq 0
\end{aligned}
$$

for all $t>0$, so $B z=z$. Thus $z$ is a common fixed point of $A$ and $B$.

Let $w$ be another common fixed point of $A$ and $B$. Then using (i), we have

$$
\begin{aligned}
& M^{2}(z, w, k t)+a M(z, w, 2 k t) \\
& \quad \geq[p+q M(z, w, t)] M(z, w, 2 k t), \\
& N^{2}(z, w, k t) \leq q N(z, w, t) N(z, w, 2 k t)
\end{aligned}
$$

and

$$
\begin{aligned}
& M(z, w, t) M(z, w, 2 k t)+a M(z, w, 2 k t) \\
& \quad \geq[p+q M(z, w, t)] M(z, w, 2 k t), \\
& N(z, w, t) N(z, w, 2 k t) \leq q N(z, w, t) N(z, w, 2 k t)
\end{aligned}
$$

Thus, it follows that

$$
M(z, w, t) \geq \frac{p-a}{1-q}=1, \quad N(z, w, t) \leq 0
$$

for all $t>0$, so $z=w$. Hence $A$ and $B$ have a unique common fixed point in $X$.

Theorem 4.2. Let $A, B, C$ and $D$ be self mappings of a complete intuitionistic fuzzy metric space $X$ satisfying

(i) $A(X) \subseteq D(X), B(X) \subseteq C(X)$,

(ii) There exists a constant $k \in(0,1)$ such that

$$
\begin{aligned}
& M^{2}(A x, B y, k t) *[M(C x, A x, k t) M(D y, B y, k t)] \\
& * M^{2}(D y, B y, k t)+a M(D y, B y, k t) M(C x, B y, 2 k t) \\
& \geq[p M(C x, A x, t)+q M(C x, D y, t)] M(C x, B y, 2 k t), \\
& N^{2}(A x, B y, k t) \diamond[N(C x, A x, k t) N(D y, B y, k t)] \\
& \diamond N^{2}(D y, B y, k t)+a N(D y, B y, k t) N(C x, B y, 2 k t) \\
& \leq[p N(C x, A x, t)+q N(C x, D y, t)] N(C x, B y, 2 k t)
\end{aligned}
$$

for every $x, y \in X$ and $t>0$, where $0<p, q<1$, $0 \leq a<1$ such that $p+q-a=1$,

(iii) The pairs $(A, C)$ and $(B, D)$ are weak compatible of type $(\gamma)$.

Then $A, B, C$ and $D$ have a unique common fixed point in $X$.

Proof. Let $x_{0} \in X$ be an arbitrary point. Since $A(X) \subseteq$ $D(X)$ and $B(X) \subseteq C(X)$, there exist $x_{1}, x_{2} \in X$ such that $A x_{0}=D x_{1}=y_{1}, B x_{1}=C x_{2}=y_{2}$. Because we can construct the sequences $\left\{x_{n}\right\},\left\{y_{n}\right\} \subset X$ such that $y_{2 n+1}=A x_{2 n}=D x_{2 n+1}, y_{2 n+2}=B x_{2 n+1}=C x_{2 n+2}$ for $n=0,1,2, \cdots$, we prove $\left\{y_{n}\right\}$ is a Cauchy sequence. For $x=x_{2 n}, y=x_{2 n+1}$ by (ii), we have

$$
\begin{aligned}
& M^{2}\left(A x_{2 n}, B x_{2 n+1}, k t\right) \\
& *\left[M\left(C x_{2 n}, A x_{2 n}, k t\right) M\left(D x_{2 n+1}, B x_{2 n+1}, k t\right)\right] \\
& * M^{2}\left(D x_{2 n+1}, B x_{2 n+1}, k t\right) \\
& +a M\left(D x_{2 n+1}, B x_{2 n+1}, k t\right) M\left(C x_{2 n}, B x_{2 n+1}, 2 k t\right) \\
& \geq\left[p M\left(C x_{2 n}, A x_{2 n}, t\right)+q M\left(C x_{2 n}, D x_{2 n+1}, t\right)\right] \\
& \times M\left(C x_{2 n}, B x_{2 n+1}, 2 k t\right), \\
& N^{2}\left(A x_{2 n}, B x_{2 n+1}, k t\right) \\
& \diamond\left[N\left(C x_{2 n}, A x_{2 n}, k t\right) N\left(D x_{2 n+1}, B x_{2 n+1}, k t\right)\right] \\
& \diamond N^{2}\left(D x_{2 n+1}, B x_{2 n+1}, k t\right) \\
& +a N\left(D x_{2 n+1}, B x_{2 n+1}, k t\right) N\left(C x_{2 n}, B x_{2 n+1}, 2 k t\right) \\
& \leq\left[p N\left(C x_{2 n}, A x_{2 n}, t\right)+q N\left(C x_{2 n}, D x_{2 n+1}, t\right)\right] \\
& \times N\left(C x_{2 n}, B x_{2 n+1}, 2 k t\right) .
\end{aligned}
$$


Hence

$$
\begin{aligned}
& M\left(y_{2 n+1}, y_{2 n+2}, k t\right) M\left(y_{2 n}, y_{2 n+2}, 2 k t\right) \\
& +a M\left(y_{2 n+1}, y_{2 n+2}, k t\right) M\left(y_{2 n}, y_{2 n+2}, 2 k t\right) \\
& \geq(p+q) M\left(y_{2 n}, y_{2 n+1}, t\right) M\left(y_{2 n}, y_{2 n+2}, 2 k t\right), \\
& N\left(y_{2 n+1}, y_{2 n+2}, k t\right) N\left(y_{2 n}, y_{2 n+2}, 2 k t\right) \\
& +a N\left(y_{2 n+1}, y_{2 n+2}, k t\right) N\left(y_{2 n}, y_{2 n+2}, 2 k t\right) \\
& \leq(p+q) N\left(y_{2 n}, y_{2 n+1}, t\right) N\left(y_{2 n}, y_{2 n+2}, 2 k t\right) .
\end{aligned}
$$

So, we have

$$
\begin{aligned}
& M\left(y_{2 n+1}, y_{2 n+2}, k t\right) \geq M\left(y_{2 n}, y_{2 n+1}, t\right), \\
& N\left(y_{2 n+1}, y_{2 n+2}, k t\right) \leq N\left(y_{2 n}, y_{2 n+1}, t\right) .
\end{aligned}
$$

Similarly, also we have

$$
\begin{aligned}
& M\left(y_{2 n+2}, y_{2 n+3}, k t\right) \geq M\left(y_{2 n+1}, y_{2 n+2}, t\right), \\
& N\left(y_{2 n+2}, y_{2 n+3}, k t\right) \leq N\left(y_{2 n+1}, y_{2 n+2}, t\right) .
\end{aligned}
$$

For $k \in(0,1)$, if $k_{1}=\frac{1}{k}>1$ and $t=k_{1} t_{1}$, then

$$
\begin{aligned}
& M\left(y_{n}, y_{n+1}, t\right) \geq M\left(y_{n-1}, y_{n}, k_{1} t_{1}\right) \\
& \geq \cdots \geq M\left(y_{0}, y_{1}, k_{1}^{n} t_{1}\right), \\
& N\left(y_{n}, y_{n+1}, t\right) \leq N\left(y_{n-1}, y_{n}, k_{1} t_{1}\right) \\
& \leq \cdots \leq N\left(y_{0}, y_{1}, k_{1}^{n} t_{1}\right) .
\end{aligned}
$$

Thus $\left\{y_{n}\right\}$ is a Cauchy sequence and completeness of $X$, $\left\{y_{n}\right\}$ converges to $z \in X$. Hence

$$
\begin{aligned}
\lim _{n \rightarrow \infty} A x_{2 n} & =\lim _{n \rightarrow \infty} y_{2 n+1}=\lim _{n \rightarrow \infty} D x_{2 n+1} \\
& =\lim _{n \rightarrow \infty} y_{2 n+2}=\lim _{n \rightarrow \infty} B x_{2 n+1} \\
& =\lim _{n \rightarrow \infty} C x_{2 n+2}=\lim _{n \rightarrow \infty} C x_{2 n}=z .
\end{aligned}
$$

Since $A, C$ are weak compatible of type $(\gamma), A z=C z$.

Now, taking $x=z$ and $y=x_{2 n+1}$ in (ii), we have as $n \rightarrow \infty$,

$$
\begin{aligned}
& M^{2}(A z, z, k t) *[M(C z, A z, k t) M(z, z, k t)] \\
& * M^{2}(z, z, k t)+a M(z, z, k t) M(C z, z, 2 k t) \\
& \geq[p M(C z, A z, t)+q M(C z, z, t)] M(C z, z, 2 k t), \\
& N^{2}(A z, z, k t) \diamond[N(C z, A z, k t) N(z, z, k t)] \\
& \diamond N^{2}(z, z, k t)+a N(z, z, k t) N(C z, z, 2 k t) \\
& \leq[p N(C z, A z, t)+q N(C z, z, t)] N(C z, z, 2 k t) .
\end{aligned}
$$

It follows that

$$
\begin{aligned}
& M^{2}(A z, z, k t)+a M(A z, z, 2 k t) \\
& \geq[p+q M(A z, z, t)] M(A z, z, 2 k t), \\
& N^{2}(A z, z, k t) \leq q N(A z, z, t) N(A z, z, 2 k t) .
\end{aligned}
$$

Since $M(x, y, \cdot)$ is nondecreasing and $N(x, y, \cdot)$ is nonincreasing for all $x, y \in X$, we have

$M(A z, z, t) \geq \frac{p-a}{1-q}=1, \quad N(A z, z, t) \leq \frac{0}{1-q}=0$ for all $t>0$, so $A z=z$. Hence $A z=C z=z$.

Similarly, since $B, D$ are weak compatible of type $(\gamma)$, we get $B z=D z$. For taking $x=x_{2 n}$ and $y=z$ in (ii), we have as $n \rightarrow \infty$,

$$
\begin{aligned}
& M^{2}(z, B z, k t) *[M(z, z, k t) M(D z, B z, k t)] \\
& \quad * M^{2}(D z, B z, k t)+a M(D z, B z, k t) M(z, B z, 2 k t) \\
& \geq[p M(z, z, t)+q M(z, D z, t)] M(z, B z, 2 k t), \\
& N^{2}(z, B z, k t) \diamond[N(z, z, k t) N(D z, B z, k t)] \\
& \diamond N^{2}(D z, B z, k t)+a N(D z, B z, k t) N(z, B z, 2 k t) \\
& \leq[p N(z, z, t)+q N(z, D z, t)] N(z, B z, 2 k t),
\end{aligned}
$$

then

$$
\begin{aligned}
& M^{2}(z, B z, k t)+a M(z, B z, 2 k t) \\
& \geq[p+q M(z, D z, t)] M(z, B z, 2 k t), \\
& N^{2}(z, B z, k t) \leq q N(z, D z, t) N(z, B z, 2 k t)
\end{aligned}
$$

Thus it follows that

$M(z, B z, t) \geq \frac{p-a}{1-q}=1, \quad N(z, B z, t) \leq \frac{0}{1-q}=0$

for all $t>0$, so $B z=z$. hence $B z=D z=z$. Therefore $z$ is a common fixed point of $A, B, C$ and $D$.

Let $w$ be another common fixed point of $A, B, C$ and $D$. Then we have

$$
\begin{aligned}
& M^{2}(A z, B w, k t) *[M(C z, A z, k t) M(D w, B w, k t)] \\
& * M^{2}(D w, B w, k t) \\
&+a M(D w, B w, k t) M(C w, B w, 2 k t) \\
& \geq[p M(C z, A z, t) \\
&+q M(C z, D w, t)] M(C z, B w, 2 k t), \\
& N^{2}(A z, B w, k t) \diamond[N(C z, A z, k t) N(D w, B w, k t)] \diamond N^{2}(D w, B w, k t) \\
& \quad+a N(D w, B w, k t) N(C w, B w, 2 k t) \\
& \leq[p N(C z, A z, t) \\
&\quad+q N(C z, D w, t)] N(C z, B w, 2 k t) .
\end{aligned}
$$

So,

$$
\begin{aligned}
& M^{2}(z, w, k t)+a M(z, w, 2 k t) \\
& \geq[p+q M(z, w, t)] M(z, w, 2 k t), \\
& N^{2}(z, w, k t) \leq q N(z, w, t) N(z, w, 2 k t) .
\end{aligned}
$$

Therefore

$$
M(z, w, t) \geq \frac{p-a}{1-q}=1, \quad N(z, w, t) \leq \frac{0}{1-q}=0
$$

for all $t>0$, so $z=w$. hence $A, B, C$ and $D$ have unique common fixed point on $X$. 
Example 4.3. Let $(X, d)$ be the metric space with $X=$ $[0,1]$. Denote $a * b=\min \{a, b\}$ and $a \diamond b=\max \{a, b\}$ for all $a, b \in[0,1]$ and let $M_{d}, N_{d}$ be fuzzy sets on $X^{2} \times(0, \infty)$ defined as follows :

$M_{d}(x, y, t)=\frac{t}{t+d(x, y)}, N_{d}(x, y, t)=\frac{d(x, y)}{t+d(x, y)}$

Then $\left(M_{d}, N_{d}\right)$ is an intuitionistic fuzzy metric on $X$ and $\left(X, M_{d}, N_{d}, *, \diamond\right)$ is an intuitionistic fuzzy metric space. Define self mappings $A, B, C$ and $D$ by

$$
\begin{aligned}
& A(X) \quad=1 \\
& B(X) \quad=1 \\
& C(X)= \begin{cases}1 & \text { if } x \text { is rational } \\
0 & \mathrm{x} \text { is irrational }\end{cases} \\
& D(X) \quad=\frac{x+1}{2} .
\end{aligned}
$$

If we define $\left\{x_{n}\right\} \subset X$ by $x_{n}=1-\frac{1}{n}$, then we have for $\lim _{n \rightarrow \infty} A x_{n}=\lim _{n \rightarrow \infty} S x_{n}=1$ and $A 1=1=C 1$,

$$
\begin{aligned}
& \lim _{n \rightarrow \infty} M\left(C A x_{n}, 1, t\right) \leq M(A 1,1, t)=1, \\
& \lim _{n \rightarrow \infty} N\left(C A x_{n}, 1, t\right) \geq N(A 1,1, t)=0 .
\end{aligned}
$$

Also, for $\lim _{n \rightarrow \infty} B x_{n}=\lim _{n \rightarrow \infty} T x_{n}=1$ and $B 1=$ $1=D 1$,

$$
\begin{aligned}
& \lim _{n \rightarrow \infty} M\left(D B x_{n}, 1, t\right) \leq M(B 1,1, t)=1, \\
& \lim _{n \rightarrow \infty} N\left(D B x_{n}, 1, t\right) \geq N(B 1,1, t)=0 .
\end{aligned}
$$

Therefore, $(A, C)$ and $(B, D)$ are weak compatible of $\operatorname{type}(\gamma)$. Then all the conditions of Theorem 4.2 are satisfied and 1 is a unique common fixed point of $A, B, C$ and $D$ on $X$.

\section{References}

[1] J.X. Fang, "On fixed point theorems in fuzzy metric spaces," Fuzzy Sets and Systems, vol. 46, pp. 107113, 1992.

[2] A. George, P. Veeramani, "On some results in fuzzy metric spaces," Fuzzy Sets and Systems, vol. 64, pp. 395-399, 1994.

[3] O. Kaleva, S. Seikkala, "On fuzzy metric spaces," Fuzzy Sets and Systems, vol. 12, pp. 215-229, 1984.
[4] S. Kutukcu, D. Turkoglu, C. Yildiz, "Common fixed points of compatible maps of type $(\beta)$ on fuzzy metric spaces," Commun. Korean math. Soc., vol. 21, no. 1, pp. 89-100, 2006.

[5] J,H. Park, "Intuitionistic fuzzy metric spaces," Chaos Solitons \& Fractals, vol. 22, no. 5, pp. 1039-1046, 2004.

[6] J.S. Park, "On some results for five mappings using compatibility of type $(\alpha)$ in intuitionistic fuzzy metric space," inpress.

[7] J.S. Park, S.Y. Kim, "Common fixed point theorem and example in intuitionistic fuzzy metric space," K.I.I.S., vol. 18, no. 4, pp. 524-552, 2008.

[8] J.S. Park, Y.C. Kwun, "Some fixed point theorems in the intuitionistic fuzzy metric spaces," F.J.M.S., vol. 24, no. 2, pp. 227-239, 2007.

[9] J.S. Park, Y.C. Kwun, J.H. Park, "A fixed point theorem in the intuitionistic fuzzy metric spaces," F.J.M.S., vol. 16, no. 2, pp. 137-149, 2005.

[10] J.S. Park, Y.C. Kwun, J.H. Park, "Some results and example for compatible maps of type $(\beta)$ on the intuitionistic fuzzy metric spaces," inpress.

[11] B. Schweizer, A. Sklar, "Statistical metric space," Pacific J. Math., vol. 10, pp. 314-334, 1960.

[12] S. Sedghi, D. Turkoglu, N. Shobe, "Common fixed point of compatible maps of type $(\gamma)$ on complete fuzzy metric spaces," Commun. Korean math. Soc., vol. 24, no. 4, pp. 581-594, 2009.

[13] L.A. Zadeh, "Fuzzy sets," Inform. and Control, vol. 8, pp. 338-353, 1965.

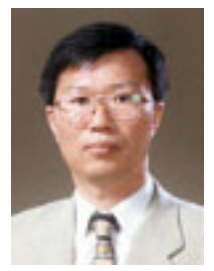

\section{Jong Seo Park}

Professor of Chinju National University of Education Research Area: Fuzzy mathematics, Fuzzy fixed point theory, Fuzzy differential equation

E-mail : parkjs@cue.ac.kr 